\title{
A new approach in the assessment of motor activity in Parkinson's disease
}

\author{
J J van Hilten, H A M Middelkoop, G A Kerkhof, R A C Roos
}

\begin{abstract}
Motor activity was recorded continuously with a wrist-worn activity monitor for almost six days in nine patients with different predominant manifestations of Parkinson's disease and 10 normal subjects. The indices of diurnal motor activity decreased with the progressive worsening of hypokinesia and rigidity. With this monitor and a simple diary it was possible to determine the contribution of a moderate resting tremor and choreiform dyskinesias to the motor activity level, and to monitor their variability.
\end{abstract}

In the clinical assessment of motor symptoms in Parkinson's disease (PD), both simple motor tests and complex neurophysiological recordings are used. ${ }^{12}$ These tests are stressful for the patient, time-consuming, costly, and assess only the momentary extent of the different motor symptoms, which renders them particularly susceptible to the influence of stress. The reliability of functional motor assessment in PD is therefore seriously hampered by fluctuations in severity of symptoms. In addition, diaries may be used, in which the patient records the duration and severity of motor symptoms. However, these diaries are cumbersome and usually not completed.

There is a need for an objective continuous, measurement procedure of motor activity which does not interfere with daily activities. Various wrist-worn activity monitors have been introduced and validated in sleep, epidemiological and behavioural studies. ${ }^{3-8}$ The activity monitor promises to be an excellent tool for the type of recording needed. However, it cannot discriminate motor activity caused by volitional movements, tremor or dyskinesias. Furthermore, with activity monitoring diurnal immobility periods due to hypokinesia are indistinguishable from naps. Thus depending on the monitor design, that is, sensitivity to a certain frequency range, this method relies partly on the traditional assessment of Parkinsonian symptoms and the keeping of a simple diary in which tremor and/or dyskinesia as well as daytime naps and sleep are indicated. We describe our preliminary results with the activity monitor.

\section{Patients and methods}

Nine patients (six males and three females) with idiopathic Parkinson's disease [age 44 80 , (mean 68) years] were compared with ten healthy subjects [five males and five females, age 55-80 (mean 70) years]. Five PD patients (patients 1-5) are characterised by hypokinesia and rigidity and show a stable levodopa response. Two PD patients (patients 6 and 7) also had a severe resting tremor. Two PD patients (patients 8 and 9) with young-onset had severe on-off fluctuations with choreiform dyskinesias. Clinical data are presented in table 1. Supplementary to levodopa patients received other anti-Parkinsonian medications, including trihexyphenidyl hydrochloride (patient 5), bromocriptine (patients 5, 6), and lisuride (patients 8, 9). Patients with a MiniMental State Examination score below 24 were excluded. ${ }^{9}$ All patients were clinically assessed by the Hoehn and Yahr and Unified Parkinson's Disease Rating Scale (UPDRS). ${ }^{1011}$ The score of the motor examination (UPDRS) and presence and severity of a resting tremor on the nondominant side are noted separately (table 1 ).

In all subjects motor activity was recorded by means of a monitor, worn on the nondominant wrist from Monday $7 \mathrm{pm}$ until Sunday $11 \mathrm{am}$. Subjects were asked to maintain their habitual 24 hour pattern of activities, and to remove the monitor only when taking a bath. During the recording period all subjects kept a $\log$ in which they recorded the time they switched off the light to
The State University, Leiden, The Netherlands Department of Physiology H A M Middelkoop G A Kerkhof Correspondence to: Dr J J van Hilten, Department of Neurology, Academic Hospital PO Box 9600, 2300 RC Leiden, The Netherlands Received 10 October 1990 and in final revised form 18 February 1991.
Table 1 Clinical characteristics of nine patients with Parkinson's disease

\begin{tabular}{|c|c|c|c|c|c|c|c|c|c|}
\hline Patient number & 1 & 2 & 3 & 4 & 5 & 6 & 7 & 8 & 9 \\
\hline $\begin{array}{l}\text { Age of onset (years) } \\
\text { Duration of illness (years) } \\
\text { Levodopa treatment (years) } \\
\text { Average dosage levodopa (g/day) } \\
\text { Hoehn and Yahr (off) } \\
\text { UPDRS total (off) } \\
\text { UPDRS motor examination score } \\
\text { Tremor sub-score }\end{array}$ & $\begin{array}{l}64 \\
0 \cdot 8 \\
0 \\
2 \\
23 \\
15 \\
0\end{array}$ & $\begin{array}{c}76 \\
2 \\
0 \cdot 5 \\
0 \cdot 4 \\
3 \\
35 \\
19 \\
0\end{array}$ & $\begin{array}{l}67 \\
5 \\
3 \\
0 \cdot 25 \\
3 \\
34 \\
19 \\
0\end{array}$ & $\begin{array}{r}68 \\
3 \\
0 \\
2 \\
44 \\
28 \\
0\end{array}$ & $\begin{array}{c}61 \\
13 \\
3 \\
0 \cdot 3 \\
4 \\
65 \\
33 \\
0\end{array}$ & $\begin{array}{c}70 \\
10 \\
10 \\
0 \cdot 4 \\
3 \\
53 \\
32 \\
3\end{array}$ & $\begin{array}{c}52 \\
13 \\
7 \\
0 \cdot 4 \\
4 \\
61 \\
28 \\
3\end{array}$ & $\begin{array}{c}37 \\
7 \\
6 \\
1 \cdot 1 \\
4 \\
67 \\
36 \\
0\end{array}$ & $\begin{array}{l}49 \\
15 \\
15 \\
1 \cdot 75 \\
4 \\
74 \\
46 \\
0\end{array}$ \\
\hline
\end{tabular}

UPDRS = the Unified Parkinson's Disease Rating Scale 

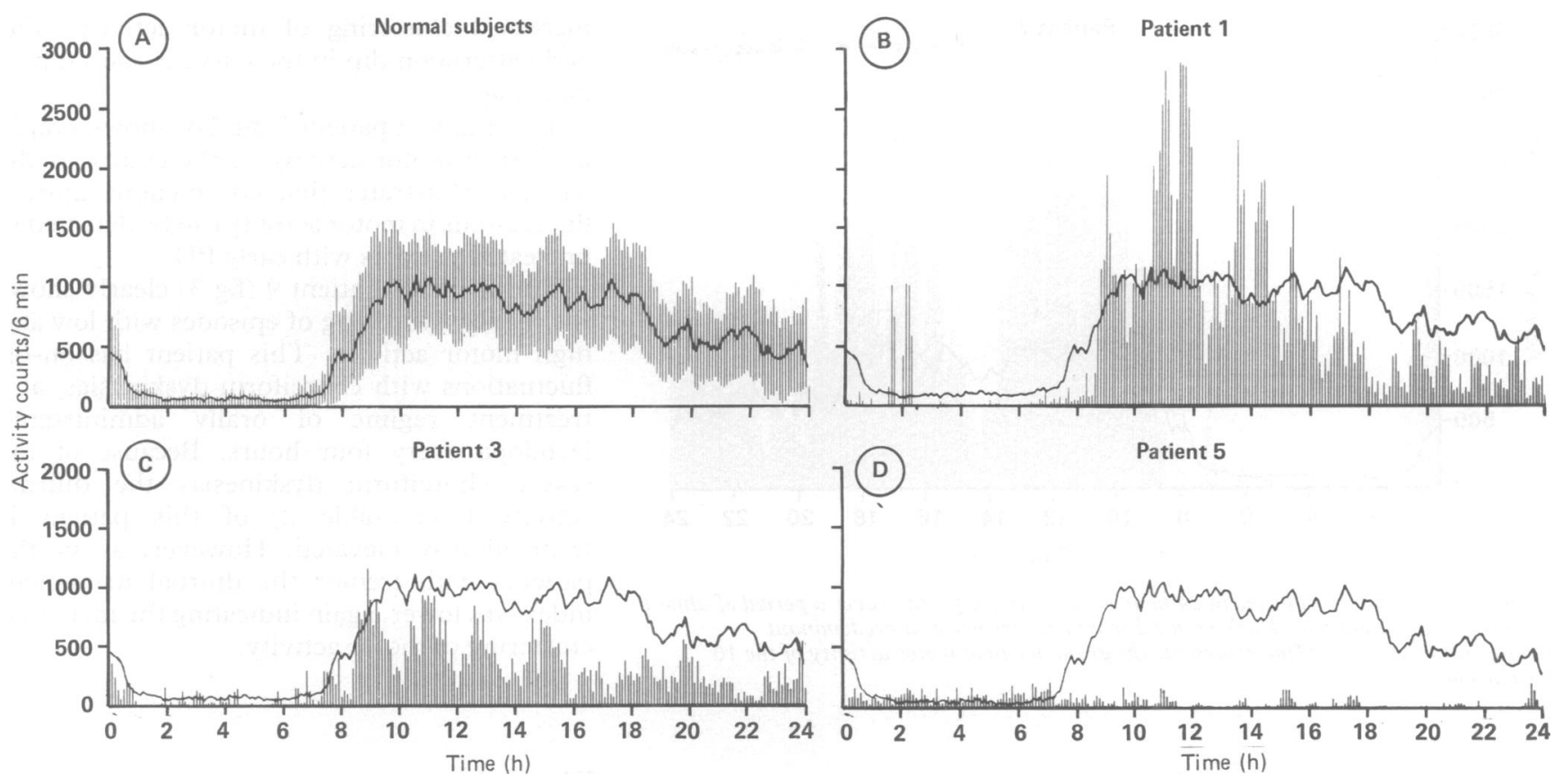

Figure $1 A$ Graph showing the 24-hour profile of the grand mean $\pm 1 S D$ of motor activity of 10 normal subjects over a period of almost six days. $B-D$ Graphs of Parkinsonian patients 1,3 and 5 showing the gradual decrease of the mean diurnal motor activity (over a period of almost six days), associated with the progression of the degree of hypokinesia and rigidity. Additionally, in patient 5 an inversion of the sleep-wake cycle is noted. The solid line represents the grand mean of motor activity of the 10 normal subjects.

go to sleep, the time of final awakening, any naps, and the time the monitor was removed when taking a bath.

The characteristics of the monitor (Gaehwiler Electronic, $\mathrm{CH}-8634$ Hombrechtikon) used in this study have been described previously. ${ }^{3}$ The device counts the occurrence of supra-threshold motor activity (accelerations $>0.1 \mathrm{~g}$ with filtering of the analog sensor signal by a bandpass filter of $0 \cdot 25-3 \mathrm{~Hz}$ ) over 30-second-epochs, and stores the resulting sum as a 1-byte value in a 32 kbyte solid state memory. After completion of the recording, the data were read out on computer and analysed further.

For each recording period the following parameters of motor activity were calculated ("diurnal" is defined as the time from final awakening to lights-out): 1) The diurnal activity level (DAL), expressed as the mean number of counts per 30 seconds calculated over all days; 2) The diurnal movement index (DMI), calculated as the mean over all days. The diurnal movement index of each day is the number of 30-second-epochs with any movement (activity count $>0$ ) expressed as percentage of 30-second-epochs comprising the diurnal period. In contrast with the DAL, the DMI reflects the degree of clustering of activity and inactivity; that is, a relatively small value indicates that motor activity only occurs during a restricted part(s) of the day; 3) The variability of the DAL and DMI during the recording period, expressed as the coefficient of variation (CV); CV-DAL and CV-DMI, respectively.

Because the graphics software (Harvard graphics) allows for only $240 \mathrm{X}$-axis values, the graphs show activity counts per six minutes which are obtained by adding the counts of 12 successive 30 -second-epochs.

\section{Results}

Normal subjects

A 24 hour plot of the overall mean (SD) values of the monitor counts for the normal subjects is presented in fig 1A. Three levels of diurnal activity may be observed: 1$)$ the night $(0.30$ am-8 am), with the lowest level of motor activity; 2) the day (8 am-6.30 pm), which shows a gradual increase of motor activity between 8-9 am and a more stable level with the highest values between 10 am and $6.30 \mathrm{pm}$; $3)$ the evening (6.30 pm-0.30 am), which shows an intermediate level of motor activity with a gradual decline. Table 2 gives the values of the various parameters of motor activity.

Table 2 Diurnal motor activity characteristics of nine patients with Parkinson's disease and mean values of 10 normal subjects. Motor activity expressed as activity counts/30 seconds

\begin{tabular}{|c|c|c|c|c|c|c|c|c|c|c|}
\hline & \multirow[b]{2}{*}{ Normal subjects } & \multicolumn{5}{|c|}{$\begin{array}{l}\text { Parkinsonian patients with } \\
\text { Hypokinesia and rigidity }\end{array}$} & \multicolumn{2}{|c|}{ Resting tremor } & \multicolumn{2}{|c|}{ Response fluctuation } \\
\hline & & 1 & 2 & 3 & 4 & 5 & 6 & 7 & 8 & 9 \\
\hline $\begin{array}{l}\text { DAL } \\
\text { CV-DAL (\%) } \\
\text { DMI (\%) } \\
\text { CV-DMI (\%) }\end{array}$ & $\begin{array}{r}75 \\
17 \\
85 \\
7\end{array}$ & $\begin{array}{l}55 \\
44 \\
66 \\
18\end{array}$ & $\begin{array}{l}38 \\
38 \\
67 \\
11\end{array}$ & $\begin{array}{l}28 \\
29 \\
59 \\
15\end{array}$ & $\begin{array}{l}10 \\
27 \\
49 \\
16\end{array}$ & $\begin{array}{r}3 \\
79 \\
19 \\
79\end{array}$ & $\begin{array}{r}77 \\
19 \\
67 \\
8\end{array}$ & $\begin{array}{r}125 \\
31 \\
75 \\
15\end{array}$ & $\begin{array}{l}50 \\
22 \\
52 \\
15\end{array}$ & $\begin{array}{r}105 \\
9 \\
62 \\
27\end{array}$ \\
\hline
\end{tabular}

DAL = diurnal activity level, DMI = diurnal movement index, $\mathrm{CV}=$ coefficient of variation. 


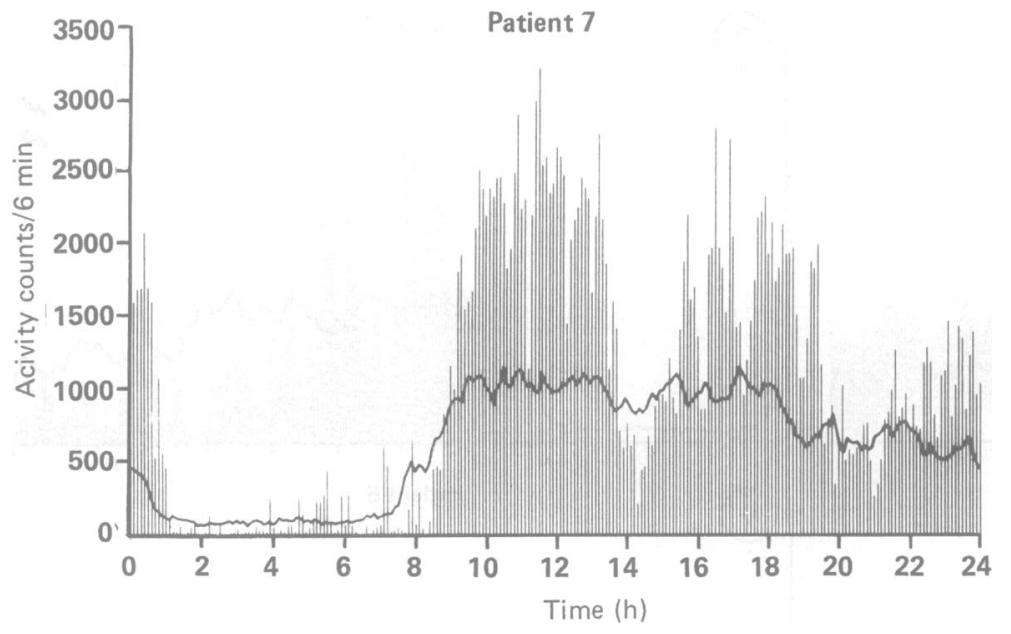

Figure 2 (patient 7). The mean 24 hour motor activity profile (over a period of almost six days) of a patient with Parkinson's disease with tremor as a predominant manifestation. The solid line represents the grand mean of motor activity of the 10 normal subjects. increased clustering of motor activity. The early afternoon dip in the curve is caused by a daily nap.

The graph of patient 1 (fig 1B) shows a rapid decline of motor activity in the course of the day and illustrates that conspicuous diurnal fluctuations in motor activity can be detected in untreated patients with early PD.

The graph of patient 9 (fig 3) clearly shows the regular switching of episodes with low and high motor activity. This patient has on-off fluctuations with choreiform dyskinesias, at a treatment regime of orally administered levodopa every four hours. Because of the severe choreiform dyskinesias the diurnal activity level (table 2) of this patient is tremendously elevated. However, as in the patients with tremor the diurnal movement index was lower, again indicating the increased clustering of motor activity.

\section{Discussion}

Parkinsonian patients

The graph of patients 1,3 and 5 (fig $1 \mathrm{~B}-\mathrm{D}$ ) reflect the decrease of motor activity associated with the increase of hypokinesia and rigidity as measured by the UPDRS motor examination score. Patients 1-5 exhibited lower values of both indices of diurnal motor activity (table 2 ). Moreover, the diurnal activity levels showed an inverse relationship to the severity of PD as measured by the total and motor examination scores of the UPDRS.

The impact of tremor upon the graph (fig 2) and the parameters (table 2) derived from it, can be estimated by comparing the results of patients 6 and 7 with patient 4 and 5 who had almost similar values of the motor examination. The presence of the tremor adds to the activity recording, to such an extent that the diurnal activity level of patient 6 resembles that of normal subjects, while the diurnal activity level of patient 7 is elevated. For both patients, however, the diurnal movement index was consistently lower, indicating a relatively

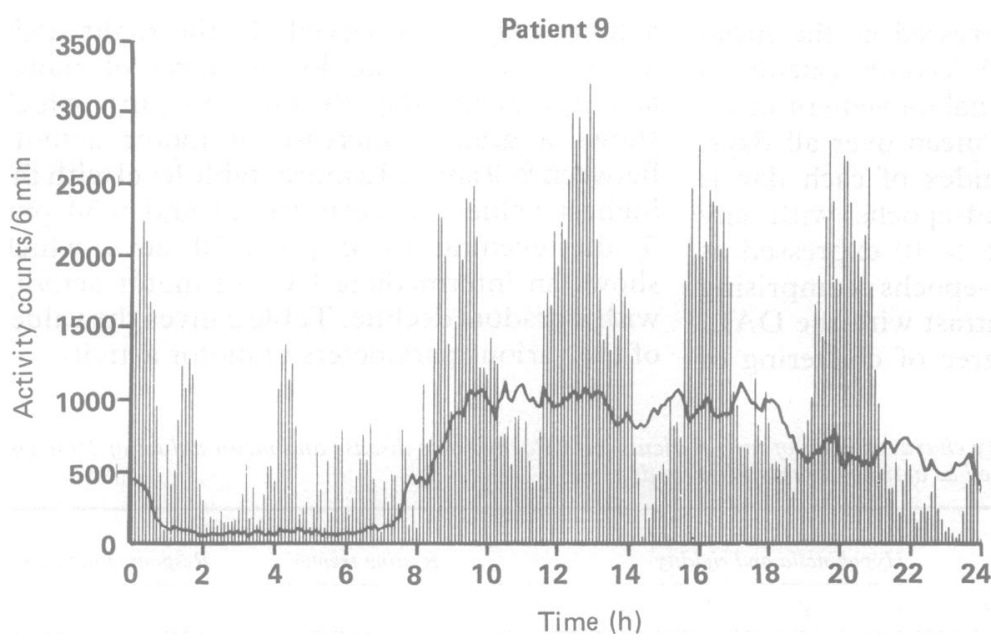

Figure 3 (patient 9). The mean 24 hour motor activity profile (over a period of almost six days) of a patient with Parkinson's disease suffering from on-off fluctuations with choreiform dyskinesias. Treatment regime of levodopa administered orally every four hours. The solid line represents the grand mean of motor activity of the 10 normal subjects.
The advantages of the monitor largely derive from its potential to record unrestrained motor activity for several days continuously, not influenced by stress such as visiting the doctor.

Our results show that each of the features of PD may contribute to the motor activity status as measured by the monitor. With the progression of hypokinesia and rigidity, the diurnal indices of motor activity decrease. On the other hand, the effects of resting tremor and choreiform dyskinesias are shown by the combined presence of a normal or elevated diurnal activity level associated with a lower diurnal movement index, indicating a relative clustering of motor activity.

In normal subjects voluntary movements do not occur at a rate greater than $200 /$ minute $(3 \cdot 3$ $\mathrm{Hz}){ }^{12}$ Because of hypokinesia and rigidity this rate will obviously be slower in patients with PD. However, in Parkinsonian patients with a predominant resting tremor, a monitor design which is specifically responsive to the frequency characteristics of the resting tremor $(4-6 \mathrm{~Hz})$ would be more desirable. In this study we were interested in whether the different motor features of PD could be monitored with the same monitor design during a continuous recording. Our results show that with the monitor design used it is possible to determine the extent to which a resting tremor which is clinically judged as moderate in amplitude and present most of the time, contributes to the motor activity level, and of its variability.

Because of the variability of symptom intensity, the assessment of the effectiveness of therapeutic interventions in PD is difficult. This applies to every stage of PD but particularly in the case of response fluctuations. The strength of this method is that the time of day effects of the on-off fluctuations can be studied, and that the amount of "off-time" can be quantified. However, the monitor data are useful if interpreted with the information obtained by a diary. Without a diary it is impossible to distinguish between the various sources of decreased (naps or severe hypo- 
kinesia) or elevated (tremor or chorea) motor activity.

In conclusion, despite some disadvantages the monitor is a welcome adjunct to the more subjective assessments of PD because of its simplicity and practicality in all settings. The monitor may prove to be of great value for studies of the disease course and response to new drug regimes.

This study was supported in part by a grant from Merck Sharp and Dohme, The Netherlands.

1 Marsden CD, Schachter M. Assessment of extrapyramidal disorders. Br J Pharmac 1981;11:129-51.

2 Lakke JWPF. Assessment and measurement. In: Stern G, ed. Parkinson's disease. London: Chapman and Hall Medical, 1990:467-91.

3 Borbely AA. New techniques for the analysis of the human sleep-wake cycle. Brain Dev 1986;8:482-8.

4 Laporte RDE, Kuller LH, Kupper DJ, McPartland RJ, Matthews G, Caspersen C. An objective measure of physical activity for epidemiological research. $A m \mathrm{~J}$ Epidemiology 1979;109:158-68.

5 Renfrew JW, Moore AM, Grady C, Robertson-Tchabo EA, Cutler NR, Rapoport SI. A method for measuring arm movements in man under ambulatory conditions. movements in man under

6 Renfrew JW, Pettigrew KD, Rapoport SI. Motor activity and sleep as a function of age in healthy men. Physiol Behavior 1987;41:627-34.

7 Webster JB, Kripke DF, Messin S, Mullaney DJ, Wyborney G. An activity-based sleep monitor system for ambulatory use. Sleep 1982;5:389-99.

8 Aubert-Tulkens GA, Rijckevorsel KH, Rodenstein DO. Ambulatory evaluation of sleep disturbance and therapeutic effects in sleep apnoe syndrome by wrist activity monitoring. Am Rev Respir Dis 1987;136:851-6.

9 Folstein M, Folstein S, McHugh PJ. "Mini-mental State": a practical method for grading the cognitive state of patients practical method for grading the cognitive state of patie

10 for the clinician. J Psychiatric Res 1975;12:189-98. and mortality. Neurology 1967;17:427-42.

11 Fahn S, Elton RL. Unified Parkinson's Disease Rating Scale. In: Fahn S, et al, eds. Recent developments in Parkinson's disease, vol 2. New Jersey, MacMillan Healthcare information, 1987:153-63.

12 Redmond DP, Hegge FW. Observations on the design and specification of a wrist-worn human activity monitoring system. Behavior Research Methods, Instruments and Computer 1985;17:659-69. 